\title{
Successful Management of Rocuronium Induced Shock in Combination with Sugammadex and Traditional Therapy
}

\author{
Hyun Ho Seong, Nam Yung Kim, Yun Ho Yang, Ju Youn Kim, Seongsik Kang* \\ Department of Anesthesiology and Pain Medicine, Institute of Medical Sciences, Kangwon National University Hospital, \\ School of Medicine, Chuncheon, South Korea \\ * Corresponding author's email: sskang [AT] kangwon.ac.kr
}

\begin{abstract}
Anaphylactic reactions during anesthesia can have a high mortality. The most common cause of anaphylaxis during anesthesia is neuromuscular blocking agents and, even though considered intermediate risk, rocuronium is frequently involved, probably due to its greater use. We present the case of a woman with anaphylactic shock secondary to the intravenous administration of rocuronium and recovered without complications by early aggressive management combination with conventional methods and sugammadex.
\end{abstract}

\section{INTRODUCTION}

Anaphylaxis during anesthesia is a rare event that may occur in up to $1: 20,000$ cases and approximately $60 \%$ to $70 \%$ of these cases are secondary to the use of muscle relaxants, particularly succinylcholine and rocuronium[1]. Rocuronium with its rapid onset of action is one of the most commonly used safe and effective muscle relaxants for intubation and maintenance of muscle relaxation during general anesthesia. Therefore its use is increasing. The standard treatment of anaphylaxis requires immediate discontinuation of the drug, whenever possible, and the use of agents that improve cardiac performance and hemodynamic profile [1]. Sugammadex was introduced into medical practice in order to antagonize the clinical action of rocuronium via encapsulation of this muscle relaxant, and this mechanism of action may contribute positively for the treatment of anaphylaxis induced by this neuromuscular blocker [2]. The aim of this report is to describe the treatment of a case of rocuronium-induced anaphylaxis with traditional therapy and sugammadex.

\section{CASE}

A 55-year old, $62 \mathrm{~kg}, 153 \mathrm{~cm}$ woman, with no relevant medical history, was scheduled for laparoscopic hysterectomy. she had no allergic reactions to medications and food, and there was no family history of allergies. There were no abnormalities in the preoperative physical examination, hematological test, simple X-ray test and ECG. No antibiotic was administered in the preoperative period or as part of the induction sequence. The preanesthetic vital signs indicated a blood pressure (BP), pulse rate and oxygen saturation of 137/85 mmHg, $75 \mathrm{bpm}$ and 99\%, respectively. General anesthesia was induced with remifentanil and propofol, TCI(Target controlled infusion) total i.v. anesthesia(TIVA). After $40 \mathrm{mg}$ of rocuronium was given, mask ventilation was noted to be difficult. Initially we thought that difficulty in ventilation was due to opioid induced chest rigidity. Therefore, remifentanil infusion was stopped and, patient was intubated 1 minutes after rocuronium injection without difficulty. Even though successful endotracheal intubation with Glidescope laryngoscopy performed, the ventilation became increasingly difficult with wheezing sound in both lung field and the oxygen saturation decreased to $73 \%$. The non invasive blood pressure and heart rate was $48 / 30 \mathrm{mmHg}, 148 \mathrm{bpm}$. Simultaneously, end-tidal $\mathrm{CO} 2(\mathrm{EtCO} 2)$ decreased $16 \mathrm{mmHg}$. Peak airway pressure increased 30mmHg. Although incremental doses of ephedrine $(10 \mathrm{mg} * 2)$ was administered, both BP and HR responded poorly to $55 / 30 \mathrm{mmHg}, 163$ bpm. The patient developed a skin erythema on the face and thorax. The diagnosis of anaphylaxis was assumed.

The initial treatment consisted of ventilation with $100 \%$ oxygen, repeated epinephrine boluses of $100 \mathrm{mcg}$ and $200 \mathrm{mcg}$, $200 \mathrm{mg}$ hydrocortisone, $5 \mathrm{mg}$ chlorpheniramine maleate and fluid loading with normal saline $1500 \mathrm{ml}$. Also, $400 \mathrm{mg}$ of sugammadex were administered, given the possibility that the condition was secondary to the use of rocuronium. With aggressive treatment, 20 minutes after rocuronium administration, the vital sign of patient was improved gradually. BP, $\mathrm{HR}$, oxygen saturation and airway pressure were checked 79/42 mmHg, $108 \mathrm{bpm}, 94 \%$ and $20 \mathrm{mmHg}$. The surgery was cancelled and the patient was allowed to emerge from anesthesia. The patient was transferred to the intensive care unit (ICU) with intubation. Laboratory test obtained from blood samples drawn 1 hour after the administration of rocuronium. Her histamine and tryptase blood level were $75.3 \mu \mathrm{mol} / \mathrm{L}$ and $47.6 \mu \mathrm{mol} / \mathrm{L}$ respectively (normal $<10 \mu \mathrm{mol} / \mathrm{L}$ ). Extubation performed 3 hours later in the ICU when she was hemodynamically stable and fully awake. She was transferred out of the ICU the next day and refused surgery and further work up about allergic study and then was discharged on the third hospital day. 


\section{DISCUSSION}

Anaphylaxis during anesthesia is a life threatening event. In the most cases of anaesthesia-related anaphylactic reactions, neuromuscular blocking drugs are known as being the causative agent, being responsible for $70 \%$ of cases, followed by reactions to latex and antibiotics.[1,3]

Among several neuromuscular blocking agents, rocuronium has been reported in a number of studies to be the most commonly related agent with increasing frequency of its use.

In our case, before anaphylaxis occurred only three drugs, propofol, remifentanil and rocuronium were administered during the induction of anesthesia. Rocuronium is presumed to be the causative agent of anaphylaxis because the injection of rocuronium initiated the increase in airway resistance and the sudden decrease in blood pressure.

Also, the episode of anaphylaxis in our patient was identified with the concurrence of erythema in face and thorax, elevated blood histamine, tryptase level. However, we could not perform an confirmatory allergen test because that patient refused any other test and operation.

Initially, the aim of anaphylactic shock treatment should be to restore both adequate cardiac output and circulatory competency. Some reports have advised use of vasopressors with alpha as well as beta stimulating properties [4,5]. Epinephrine is the most appropriate drug of choice in the management of bronchospasm and massive peripheral vasodilation. In addition to epinephrine, intravascular volume, vascular tone and cardiac output should be supported with colloid or crystalloid fluids [6]. In our case, we received a response within 20 minutes to epinephrine, normal saline $1.5 \mathrm{~L}$. Besides, antihistamines are useful prophylactically and may also prevent further histamine binding after the development of anaphylactic reactions [4][6]. Corticosteroids should be given prophylactically to diminish the airway swelling and prevent recurrence of symptoms[6].

Some recently published clinical cases have reported the value of using sugammadex in the management of rocuroniuminduced anaphylactic shock [7].

Sugammadex is a developed drug that was specifically designed for the reversal of

rocuronium-induced neuromuscular block[8]. In contrast to traditional reversal agents like pyridostigmine that work by competitive antagonism of the neuromuscular agent, sugammadex encapsulates the rocuronium molecule, negating its pharmacological effect, and essentially removing it from the circulation[8].

The potential reasons for the reversal of the hemodynamic state are unclear. it may be that the binding of the rocuronium molecule by sugammadex prevented further vasoactive mediator release and allowed the previously administered epinephrine to have increased efficacy. As the administration was also associated with reversal of the patient's neuromuscular block, it may be that the associated increase in muscle tone assisted with the restoration of venous return and cardiac output. It is also possible that the effect was purely by coincidence and that the reversal in her clinical condition was secondary to the epinephrine and fluid resuscitation that had been instituted.

\section{CONCLUSION}

The favorable pharmacokinetics of rocuronium have led to an increased frequency of use during general anesthesia. We would advise anesthetists using rocuronium to be aware of the possibility of rocuronium induced anaphylaxis.

If rocuronium induced anaphylaxic shock occurs, early treatment with vasoactive drugs and fluids is needed, and if it is not effective, sugammadex can be considered.

\section{REFERENCES}

1. McDonnell NJ, Pavy TJ, Green LK et al. - Sugammadex in the management of rocuronium-induced anaphylaxis. Br J Anaesth, 2011;106:199-201.

2. Jones PM, Turkstra TP - Mitigation of rocuronium-induced anaphylaxis by sugammadex: the great unknown. Anaesthesia, 2010;65:89-90

3. Mertes PM, Laxenaire MC, Alla F. Anaphylactic and anaphylactoid reactions occurring during anesthesia in France in 1999-2000. Anesthesiology 2003; 99: 536-45

4. Jakubowski MS, Barnard DE. Anaphylactic shock during operation for hydatid disease. Anesthesiology 1971; 34: 197-9.

5. CurrieM, Kerridge RK, BaconAK,Williamson JA. Crisis management during anaesthesia: anaphylaxis and allergy. Qual Saf Health Care 2005; 14: e19.

6. Mali S, Jambure R. Anaphyllaxis management: Current concepts. Anesth Essays Res. 2012 Jul-Dec;6(2):11523. doi: 10.4103/0259-1162.108284. Review.

7. B. Plaud A new option for the treatment of anaphylaxis linked to steroidal neuromuscular blockers: How much should we grant to case reports? Can J Anaesth, 61 (2014), pp. 511-518

8. Naguib M. Sugammadex: another milestone in clinical neuromuscular pharmacology. Anesth Analg 2007; 104: $575-81$ 